\title{
INVESTIGATION OF CHROMATICITY SHARING AT THE CORNELL ELECTRON STORAGE RING
}

\author{
S. Henderson, R. Littauer, B. McDaniel, T. Pelaia, R. Talman, E. Young, \\ Laboratory of Nuclear Studies, Cornell University, Ithaca, NY 14853*
}

\section{Abstract}

A potential advantage of the "Möbius" scheme, or any other strongly coupled lattice, is that the normal-mode oscillations are damped at rates which depend on both the horizontal and vertical chromaticities. This opens up the possibility of maintaining adequate damping even for quite negative chromaticity in one plane, provided the other is appropriately increased to compensate. This "chromaticity sharing" has been observed at CESR by introducing resonant coupling with weak skew quadrupoles and observing the coherent damping rates of shock-excited betatron oscillations.

\section{INTRODUCTION}

Chromaticity compensation is required in high-energy storage rings to avoid instability due to the head-tail (H-T) effect $[1,2]$. We define the chromaticity of a lattice as

$$
Q^{\prime}=d Q / d \delta
$$

where $Q$ is the betatron tune and $\delta$ is the fractional deviation from nominal momentum. The natural chromaticity of a lattice is negative, which produces a positive growth rate $\alpha$ in the lowest (dipole, $m=0$ ) H-T mode; damping $(\alpha \leq 0)$ requires $Q^{\prime} \geq 0$. Ordinarily, sextupoles are introduced in the lattice to make $Q^{\prime} \geq 0$ in both the $x$ and $y$ planes of oscillation. ${ }^{1}$

A sextupole produces opposite chromaticity changes in $x$ and $y$. Thus at least two families of sextupoles are required, so placed as to yield a net positive correction in both planes. To the extent that the $x$ and $y$ sextupoles have opposing effects, their individual strengths can become relatively large, particularly for the $y$ sextupoles, which are less effective because they tend to be in regions of small dispersion. Strong sextupoles reduce the dynamic aperture of the lattice.

A potential advantage of the Möbius scheme proposed by Talman [3] is that normal-mode oscillations, containing both $x$ and $y$ components, should be damped at a rate that depends on both $\alpha_{x}$ and $\alpha_{y}$, an effect we call chromaticity sharing. It promises to maintain $\mathrm{H}-\mathrm{T}$ stability with a less restrictive choice of sextupoles; instead of both $Q_{x}^{\prime} \geq 0$ and $Q_{y}^{\prime} \geq 0$, the criterion becomes $Q_{x}^{\prime} W_{x}+Q_{y}^{\prime} W_{y} \geq 0$, where $W_{x}, W_{y}$ are factors describing the relative magnitudes of the $x$ and $y$ wake fields affecting the bunch.

\footnotetext{
${ }^{*}$ Work supported by the National Science Foundation

${ }^{1}$ Because $Q^{\prime}>0$ produces a positive growth rate in the higher $\mathrm{H}$ T modes, $Q^{\prime}$ is generally set to zero or only slightly positive. Then the higher modes, with smaller form factors and wake-field coefficients, are stabilized adequately by radiation and Landau damping.
}

Talman [4] has generalized the two-macroparticle model of the H-T effect to include $x-y$ coupling. The two coupled modes of interest show equal growth rates of the form

$$
\alpha \propto \frac{I \hat{\delta}}{Q_{s}}\left(Q_{x}^{\prime} W_{x}+Q_{y}^{\prime} W_{y}\right)
$$

where $I$ is the bunch current, $\hat{\delta}$ is a typical peak momentum deviation, and $Q_{s}$ is the momentum-oscillation tune. (An additional term which depends on the coupling strength $S$, $\left(Q_{x}^{\prime}-Q_{y}^{\prime}\right)\left(W_{x}-W_{y}\right) \frac{S^{2} / 2}{Q_{s}^{2}-S^{2}}$, small everywhere except near $S \simeq Q_{s}$, has been neglected.) In this model, constant H-T damping-the same for both coupled modes-occurs along a line in the $Q_{x}^{\prime}, Q_{y}^{\prime}$ plane of slope $-W_{x} / W_{y}$.

Alternatively, one can ascribe a chromaticity directly to each of the normal coupled modes. When the lattice is at resonance (equal fractional tunes, $q_{x}=q_{y} \equiv q_{0}$ ), turning on a coupling element splits the tunes into upper and lower normal modes: $q_{U, L}=q_{0} \pm \frac{1}{2} S$, where the tune split $S$ is a measure of the coupling strength. If $Q_{x}^{\prime} \neq Q_{y}^{\prime}$, a particle off-momentum by $\delta$ is detuned from resonance by $D=$ $\left(Q_{x}^{\prime}-Q_{y}^{\prime}\right) \delta$; the mode tunes, centered on $\bar{q}=q_{0}+\frac{1}{2}\left(Q_{x}^{\prime}+\right.$ $\left.Q_{y}^{\prime}\right) \delta$, are then separated by $\sqrt{S^{2}+D^{2}}$.

Figure 1 shows $q_{U}$ and $q_{L}$ as a function of $\delta$ for an extreme case, $Q_{x}^{\prime}=20, Q_{y}^{\prime}=-10, S=0.026$. The curves are what is measured by a tune tracker in steady-state conditions when the beam is taken off-momentum by changing the RF frequency. Particles within a CESR bunch might typically have $\delta= \pm 1.5 \sigma_{\delta} \simeq \pm 10^{-3}$, detuning them from resonance, for the case illustrated, by $D \simeq 0.01-0.02$, which is of the same order as the coupling strength $S$. (Möbius coupling will be dramatically stronger.) Clearly, if

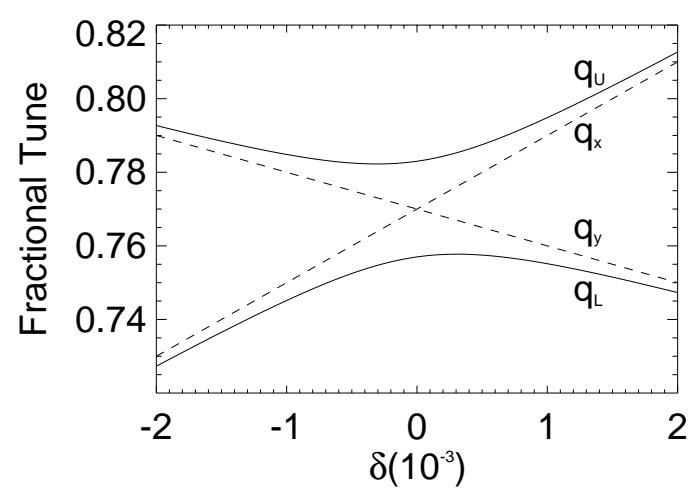

Figure 1: The dependence of the normal mode tunes on energy for $Q_{x}^{\prime}=20, Q_{y}^{\prime}=-10, S=0.026$. 
$\delta$ varies slowly enough for the modes to retain their identity $\left(S \gg Q_{s}\right)$, the chromaticity of each mode is highly nonlinear, which might be expected to lead to decoherence (Landau damping). Such effects would of course not appear in the simple two-superparticle model. On the other hand, if $\delta$ changes so rapidly as to mix the modes $\left(S \ll Q_{s}\right)$, the effective chromaticity may become that associated with the average tune $\bar{q}$, that is $\frac{1}{2}\left(Q_{x}^{\prime}+Q_{y}^{\prime}\right)$. In fact, $\delta$ oscillates at frequency $Q_{s} f_{0}$; in CESR, $Q_{s} \simeq 0.05$, comparable to the "tune" $S$ of beats between the modes.

Since tune-tracking studies do not duplicate the conditions of momentum oscillations, we concentrated on measurements of $\alpha$, the coherent damping rate of shock-excited betatron oscillations. Initially we pursued the expectations described by equation 2 .

\section{EXPERIMENTAL}

We measured the coherent damping rates by shock-exciting the beam with a fast kicker and observing the response of beam-position monitor signals on a fixed-tune spectrum analyzer (bandwidth $=2 \mathrm{kHz}$, i.e., $\Delta q \simeq 5 \times 10^{-3}$ ). The analyzer displayed the damping envelope logarithmically, allowing-after subtraction of the incoherent radiation damping rate $\alpha_{R} \simeq 38 \mathrm{~s}^{-1}$-determination of the coherent damping rate $\alpha$.

The accelerator lattice used for these experiments was optimized for studying round-beam collisions at CESR; it is described in an accompanying paper [5]. Chromaticity correction is accomplished with sextupoles in two families. $Q_{x}^{\prime}$ and $Q_{y}^{\prime}$ are independently controlled by simultaneously varying the strengths of the two families; we verified that $Q_{x}^{\prime}$ and $Q_{y}^{\prime}$ were linear functions of the sextupole strengths and that the $x$ and $y$ chromaticity controls were orthogonal. The $\mathrm{H}-\mathrm{T}$ damping rates in each plane depended linearly on the associated chromaticity over the range for which a stable beam could be maintained $(0 \leq$ $Q^{\prime} \leq 12$ ). The H-T damping coefficients (measured at $\sim 5$ $\mathrm{mA}$ beam current) were $d \alpha_{x} / d Q_{x}^{\prime}=-5.54 \mathrm{~s}^{-1} \mathrm{~mA}^{-1}$ and $d \alpha_{y} / d Q_{y}^{\prime}=-6.19 \mathrm{~s}^{-1} \mathrm{~mA}^{-1}$, indicating that the wakefield coefficients are in the ratio $W_{x} / W_{y}=0.89$.

The machine was taken to resonance (typically to within $D_{0} \leq 2 \times 10^{-3}$ ) and globally decoupled. Two skew quadrupoles placed symmetrically with respect to the interaction point were then powered to introduce the desired coupling strength $S$. In this coupled lattice, the measured normal-mode damping rates depended neither on the orientation of the kick nor of the pickup electrodes ( $x$ or $y$ ).

\section{RESULTS}

\subsection{Weak Coupling $\left(S<Q_{s}\right)$}

To check equation 2 , we started from a somewhat arbitrary point ( $Q_{x}^{\prime} \simeq 4, Q_{y}^{\prime} \simeq 0$ ) and followed contours of constant $\alpha_{U}$ or $\alpha_{L}$ in the $Q_{x}^{\prime}, Q_{y}^{\prime}$ plane. The damping rates of the two modes were generally quite different, contrary to the prediction of the two-particle model; the two con-
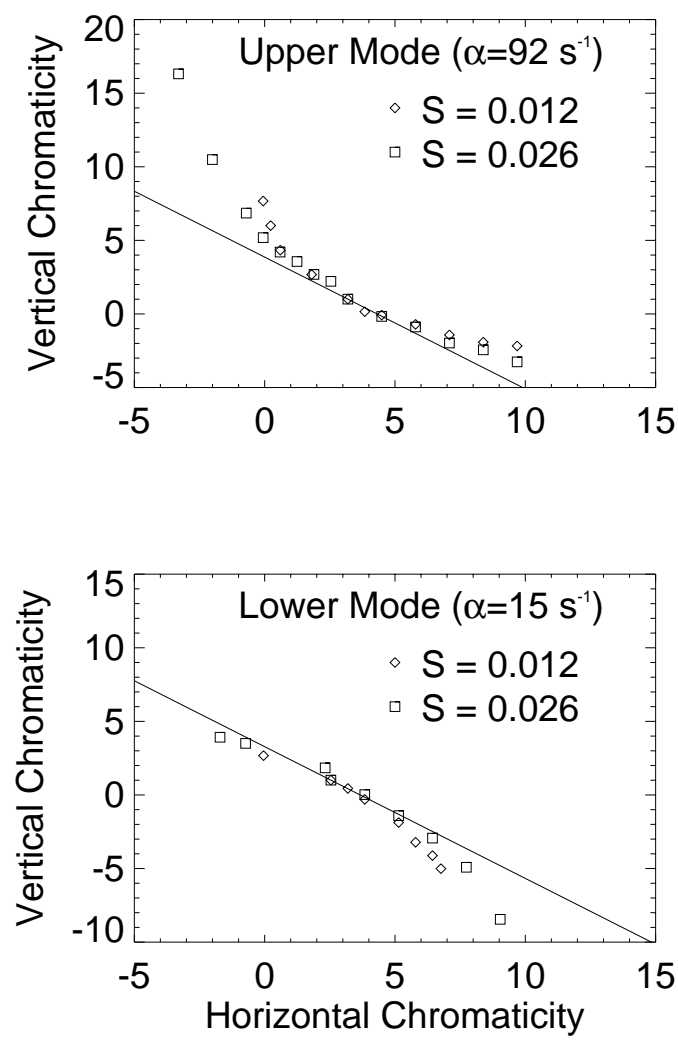

Figure 2: Contours of constant damping rate in the $Q_{x}^{\prime}, Q_{y}^{\prime}$ plane for the two coupled modes.

tours shown in figure 2 correspond to $\alpha_{U}=92 \mathrm{~s}^{-1}$ and $\alpha_{L}=15 \mathrm{~s}^{-1}$. Data are shown for two coupling strengths, $S=0.012$ and 0.026 . The range of data represents the range of $Q^{\prime}$ over which a stable beam could be maintained along these contours with this coupling.

The curves demonstrate that there is chromaticity sharing, even for relatively weak coupling. (For comparison, straight lines with the expected slope $-W_{x} / W_{y}=-0.89$ are included on the plots.) However, the contours are not straight; their curvature, opposite for $\alpha_{U}$ and $\alpha_{L}$, is only slightly reduced by stronger coupling. Most importantly, the disparity between $\alpha_{U}$ and $\alpha_{L}$ calls for explanation.

To investigate the possibility that the physical orientation of the modes in the vacuum chamber played a role, we reversed the sign of the skew quadrupoles, thus interchanging the orientation of the $\mathrm{U}$ and $\mathrm{L}$ modes. This had a systematic effect only at small $Q^{\prime}$, that is, for small damping rates $\left(\simeq 10 \mathrm{~s}^{-1}\right)$. The larger observed damping rates therefore appear to be linked to the modes themselves, not their spatial orientation. We always found $\alpha_{U}>\alpha_{L}$.

\subsection{Intermediate Coupling $\left(S \simeq Q_{s}\right)$}

Upon increasing the coupling strength to 0.039 we found it possible to maintain a stable beam over a much larger range of $Q^{\prime}$, although some conditions could not be approached 

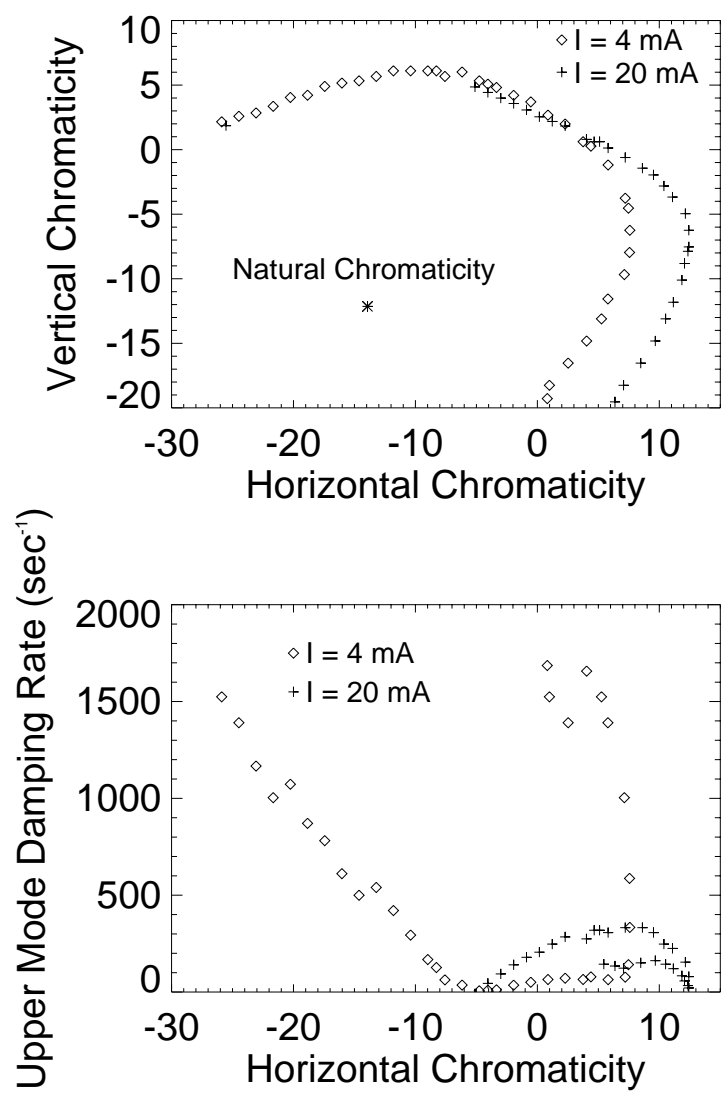

Figure 3: (Upper) Contours of constant damping rate for the lower frequency mode for coupling $S=0.039$. Contours corresponds to $\alpha_{L}=14 \mathrm{~s}^{-1}(4 \mathrm{~mA})$ and $\alpha_{L}=20$ $\mathrm{s}^{-1}(20 \mathrm{~mA})$. (Lower) Corresponding damping rate of the upper frequency mode recorded at each point of the contour.

directly by following a contour of constant $\alpha$. Figure 3 shows contours of constant $\alpha_{L}$ for two widely different beam currents. Also shown on the lower portion of the figure are the corresponding $\alpha_{U}$ recorded at each point of the contour. The striking feature is that, for highly negative $Q^{\prime}$ in either plane, stability is obtained with much smaller positive $Q^{\prime}$ in the other plane than would be called for with simple chromaticity sharing. In fact, at each end of the contour one family of sextupoles is turned off completely, leaving $Q^{\prime}$ in the corresponding plane much more negative than the natural value for the lattice $(\simeq-12)$, owing to the effect of the other sextupole family. At these extreme points, the damping rate of the upper mode is quite large.

\subsection{Qualitative Investigations}

This unexpected behavior suggests a decoherence phenomenon due to the large spread in tunes (figure 1), an idea supported by the fact that at extreme settings-for example, $Q_{x}^{\prime} \simeq 20, Q_{y}^{\prime} \simeq-20$-both modes are heavily damped $\left(\alpha_{U, L}>1000 \mathrm{~s}^{-1}\right)$ even at low beam current $(2 \mathrm{~mA})$. How- ever, though the coherent oscillation signal disappeared in times well below $1 \mathrm{~ms}$, the optical image nevertheless retained considerable enlargement for times comparable to the interval between the kicks (which occurred at $60 \mathrm{~s}^{-1}$ ). Clearly, the oscillations quickly become incoherent and individual particle amplitudes are damped only at the low radiation rate, $38 \mathrm{~s}^{-1}$.

If there is decoherence, it should contribute Landau damping independently of the beam current, to an extent determined by the tune modulation produced by momentum oscillations. That is consistent with the general shape of the constant- $\alpha$ contours at extreme $Q^{\prime}$. (An attempt to change the momentum oscillations by lowering the RF voltage from an overvoltage of $6 \times$ to $4 \times$ revealed no significant change in damping rates.)

Apparently, in the intermediate coupling regime $(S \simeq$ $Q_{s}$ ), the tune split is large enough for the modes to assert themselves; the momentum oscillations lead to a spread in tunes. The non-linear chromaticity leads naturally to decoherence; unlike the familiar case of linear chromaticity, a particle executing momentum oscillations does not return to its initial betatron phase after one synchrotron period. Furthermore, particles with different longitudinal oscillation amplitudes arrive at slightly different phases following one oscillation period, giving rise to decoherence.

\section{CONCLUSION}

The expected chromaticity sharing in a coupled lattice is confirmed; it occurs even for weak coupling. For intermediate coupling, however, damping mechanisms other than the head-tail effect appear to become dominant. These are tentatively identified as effects of decoherence, produced by the large tune modulation during momentum oscillations, and the nonlinear chromaticity of the coupled modes. Further work-especially in the Möbius regime-is planned.

\section{ACKNOWLEDGEMENTS}

We would like to thank the CESR Operations staff for their help with these experiments.

\section{REFERENCES}

[1] M. Sands, SLAC-TN-69-8, C. Pellegrini, Nuovo Cim. 64A, 477 (1969).

[2] R. Talman, NIM, 193, 423 (1982), R.D. Kohaupt, DESY 80/22 and DESY M-80/19, (1980).

[3] R. Talman, Phys. Rev. Lett., 74, 1590(1995).

[4] R. Talman, "Theory of Head-Tail Chromaticity Sharing," CBN-97-17

[5] E. Young et al, "Collisions of Resonantly Coupled Round Beams at the Cornell Electron-Positron Storage Ring (CESR)", these proceedings. 